\title{
Birth Timing and Neonatal Health ${ }^{\top}$
}

\author{
By Cristina Borra, Libertad González, and Almudena Sevilla*
}

We exploit a policy change that exogenously led to many deliveries being scheduled earlier, to assess the causal effect of scheduling birth early for nonmedical reasons on infant health beyond birth. More and more children are born before the mother spontaneously goes into labor, either through labor inductions or C-sections, which combined now surpass half of all births in the United States (Byanova 2015). Given the well-established impact of infant health on long-term outcomes (Smith 2009; Fletcher, Green, and Neidell 2010), understanding the health effects of early delivery is important to inform the design of early policy interventions, with potentially far-reaching effects.

There is limited correlational evidence in the medical literature on the relationship between gestational length and health outcomes beyond birth. Recent studies suggest that early delivery, even after 37 weeks of gestation, can carry negative health consequences for the child (Boyle et al. 2012). Recent work in economics has provided evidence on the effect of early delivery on health at birth (Schulkind and Shapiro 2014), measured via birthweight and Apgar scores, with no exploration of subsequent health outcomes later on during infancy. To our knowledge, this is the first study to provide credible causal evidence on the effect of scheduling birth early for nonmedical reasons on health outcomes after birth. Here we focus on the neonatal period, while in Borra, González, and Sevilla (2015) we explore longer-term outcomes in more detail.

\footnotetext{
* Borra: Department of Economics and Economic History, University of Seville, Ramon y Cajal 1, Seville 4018, Spain (e-mail: cborra@us.es); González: Department of Economics and Business, Universitat Pompeu Fabra, Ramon Trias Fargas, 25-27, Barcelona, 08005, Spain, and Barcelona GSE (e-mail: libertad.gonzalez@upf.edu); Sevilla: School of Business and Management, Queen Mary, University of London, Francis Bancroft Building, Mile End Road, London E1 4NS (e-mail: a.sevilla@qmul.ac.uk)

${ }^{\dagger}$ Go to http://dx.doi.org/10.1257/aer.p20161123 to visit the article page for additional materials and author disclosure statement $(\mathrm{s})$.
}

We exploit a quasi-natural experiment that (exogenously) generated an incentive to shift forward the date of a large number of births for nonmedical reasons in Spain. In May 2010 the Spanish government announced the cancellation of a generous 2,500-euro universal child benefit, in effect for children born after December 31,2010 . The intervention affected about 2,000 children due near the benefit cancellation cutoff, so that as much as 6 percent of all January 2011 births were shifted back between one and three weeks to December in order to qualify for the benefit (as we document in Borra, González, and Sevilla 2015). The shifting was driven by full-term pregnancies (at least 37 weeks of gestation at birth) and newborns above the low birthweight threshold of 2,500 grams, and we find no increase in the overall $\mathrm{C}$-section rate, only a shifting from January to December.

We use detailed, high-quality administrative data from hospital records and birth certificates for the universe of children born in Spain between 2000 and 2012, to evaluate health outcomes at, as well as after, birth. Our identification strategy relies on comparing infant health outcomes of children born near the benefit cancellation date (December 2010 and January 2011) with children born in the same dates in the surrounding years, using births in the surrounding months as controls.

We find that children born close to the benefit cancellation date suffered significantly higher hospitalization rates in the weeks following birth. We find no effect on medical conditions right at the time of birth or the week after, but we document a significant spike in hospital stays starting the second week after birth, suggesting potentially persistent health effects.

\section{Empirical Strategy}

We identify the causal effect of scheduling birth early for nonmedical reasons on infant health by comparing the health of babies born in December 2010 and January 2011 with the 
health of babies born in the same dates but in the surrounding years, using children born in other months of the year as controls. By including both December and January births in the "treated" group, we take care of potential composition effects that may confound the causal effect of bringing the birth forward. To the extent that families shifting births from January 2011 to December 2010 in order to receive the benefit tend to have healthier (less healthy) children, for reasons unrelated to the timing of birth, comparing health outcomes of children born in December 2010 with January 2011 would lead to an underestimation (overestimation) of the causal effect of earlier delivery on infant health.

We include children born in October, November, February, and March as a control group. Thus, we control for other factors, such as the business cycle or weather shocks, which may have affected the health outcomes of babies born near the end of 2010, relative to children born in the surrounding years. Our main identification assumption is thus that there was no other factor affecting the health of babies born in December 2010-January 2011 differentially with respect to babies born in the four surrounding months, other than seasonal factors, present every year.

We estimate the following specification:

$$
\begin{aligned}
H_{i t m}= & \alpha+\beta(\text { Dec2010-Jan2011 })_{i t m} \\
& +\lambda_{t}+\delta_{m}+\varepsilon_{i t m},
\end{aligned}
$$

where $H_{i t m}$ is a binary indicator for hospitalization of child $i$, born in turn-of-the-year $t$, and month $m$. We control for calendar month and turn of the year fixed effects. Results are unchanged if we include indicator variables for each month-pair (October-November, December-January, and February-March) instead of month dummies.

The main explanatory variable, Dec2010-Jan2011, takes value one for babies born close to the benefit cancellation date, i.e., in December 2010 or January 2011. The coefficient of interest, $\beta$, is thus a difference-in-differences estimate that compares hospitalization outcomes for December-January babies born in the reform period (2010-2011) with those born in the same dates in the surrounding years, using October, November, February and March births as controls.
Given that we focus on health outcomes in the 21 days following birth, while the benefit was paid with a delay of at least three weeks after delivery, the coefficient $\beta$ captures the effects of scheduling birth early, while not capturing the possible health effects of receiving the benefit. Moreover, October-November 2010 births received the benefit, while February-March 2011 ones did not, so the control months net out any potential "anticipation" effects of the benefit.

We estimate equation (1) for hospitalizations by age of the child, from birth to 21 days of age. First we include all hospital stays starting between birth and 21 days. Then we split them into three age intervals: birth hospitalizations, i.e., those originating immediately after birth; hospital stays where the child is between 1 and 7 days of age at admission, and those starting at 8 to 21 days of age.

We use four different samples for each age group, varying the window of birthdates around the benefit cancellation date. As we include births further away from the threshold, the fraction of affected babies falls, but we include children whose birthdate was potentially shifted by more. The full sample includes all infants born in the last four weeks of October, December, and February and the first four weeks of November, January, and March, for the 12 years from 2000-2001 to 2011-2012. Results are robust to including only a more recent sample of October-March births (between 2007-2008 and 2011-2012).

\section{Data}

We combine administrative data for the universe of births and hospital stays in Spain in 2000-2012, provided by the Spanish National Statistical Institute. Hospital data come from the Hospital Morbidity Survey, which records 99 percent of overnight hospital stays in Spain annually, and birth data come from official birth certificates.

Hospital records include the date of release and length of each overnight stay, as well as the age of the patient in years, months, and days. For each hospital stay, we compute the date of birth of the patient using the information on date of release and age. We then construct a count of hospitalizations in different age ranges, by date of birth. From the birth certificate data, 
we calculate the total number of children born per day. Finally, we combine both datasets, and end up with information, at the individual infant level, on exact date of birth, and a set of hospitalization indicators by age.

We select all overnight hospitalizations of children up to 21 days of age (including birth hospitalizations with a medical diagnosis), with birthdates from October to March of 2000-2001 to 2011-2012. We consider hospitalizations with a medical diagnosis, and thus exclude hospital stays for medical observation only but with no diagnosis recorded, as well as the birth hospitalization of healthy newborns. Hospital records do not provide individual identifiers, so we cannot separate the intensive from the extensive margin. The results should thus be interpreted as number of hospital stays per 100 births, and not the fraction of babies with at least one hospital stay.

Columns 1-2 in Table 1 show some descriptive statistics. We observe a total of 409,879 hospitalizations in our sample (column 1 of panel A), or almost 17 hospital stays per 100 children during the first three weeks of life (column 2). Hospitalizations are much more common right after birth, so that about 58 percent of the hospital stays in the sample are birth hospitalizations, and about another 30 percent take place between 24 hours and 7 days of age. The remaining 11 percent come from the following 14 days. Column 2 shows that almost 10 percent of all birth hospitalizations have an associated medical diagnosis, indicating some complication or medical condition detected at or right after birth. There are about 5 hospital admissions per 100 children between 1 and 7 days of age, and almost 2 per 100 children at ages between 1 and 3 weeks.

\section{Results}

Columns 3-6 in Table 1 show the regression results for hospital stays, in the full sample (panel A) as well as by age of the child in days (panel B). Each of the four columns refers to a different window of birthdates around the threshold. The first column shows the results for the sample of babies born no more than a week away from December 31, whereas the last column shows the results for the sample of children born up to four weeks away from the benefit cancellation date.
Panel A shows that children born close to the benefit cancellation date suffered unusually high hospitalization rates in their first 21 days of life. The estimated effect is significant when we include children born one, two, or three weeks before and after the cutoff. For the two-week window, we estimate a significant increase of 0.63 hospital stays for every 100 children as a result of the benefit cancellation. Given that there are about 17 hospitalizations per 100 children between birth and 21 days, this amounts to an increase in the hospitalization rate of almost 4 percent (calculated as the increase of 0.63 per 100 children, over the total number of hospital stays, 17 per 100). Note, however, that only a small fraction of the children in the sample were actually affected by the policy change, so that this should be interpreted as an "intent to treat" estimate.

Panel A also shows that the magnitude of the increase in hospitalization rates declines very slowly as we increase the window of birthdates around the threshold. Given that the fraction of affected children (those whose birthdate was moved in order to receive the benefit) decreases as we include births further away from the cutoff (as shown in Borra, González, and Sevilla 2015), this suggests that the negative health effects of early delivery were stronger for children whose birthdate was moved by more.

Panel B of Table 1 shows the results by age of the newborn at hospital admission. The first row shows that there was no significant increase in medical conditions or complications at birth as a result of the benefit cancellation, since we find no effect on the number of birth hospitalizations with a medical diagnosis, in any of the four samples. This suggests that any possible congestion effects generated by the spike in December births did not lead to a rise in the rate of complications during or right after delivery.

The second row of panel B shows that the children born close to the benefit cancellation date were not more likely to be readmitted to the hospital during their first week of life, compared with babies born in the surrounding months or years. However, the final row of Table 1 shows a significant increase in hospitalization rates for the affected cohort of babies during their second and third weeks of life. In particular, in the two-week window, there were about 0.33 additional hospital stays per 100 children as 
Table 1-The Effect of Benefit Cancellation on Infant Hospitalizations

\begin{tabular}{|c|c|c|c|c|c|c|}
\hline & \multicolumn{2}{|c|}{ Descriptives } & \multicolumn{4}{|c|}{ Regression results } \\
\hline & $\begin{array}{c}\text { Number of } \\
\text { hospitalizations } \\
\text { (1) }\end{array}$ & $\begin{array}{l}\text { Hospitalization } \\
\text { rate } \\
(2)\end{array}$ & $\begin{array}{c}+/-1 \\
\text { weeks } \\
(3)\end{array}$ & $\begin{array}{c}+/-2 \\
\text { weeks } \\
(4)\end{array}$ & $\begin{array}{c}+/-3 \\
\text { weeks } \\
(5)\end{array}$ & $\begin{array}{c}+/-4 \\
\text { weeks } \\
(6)\end{array}$ \\
\hline \multicolumn{7}{|c|}{ Panel A. Total hospitalizations (0-21 days) } \\
\hline Total hospitalizations & 409,879 & 0.1673 & $\begin{array}{l}0.0062 * \\
(0.003)\end{array}$ & $\begin{array}{l}0.0063 * * \\
(0.002)\end{array}$ & $\begin{array}{l}0.0057 * * * \\
(0.002)\end{array}$ & $\begin{array}{c}0.0024 \\
(0.002)\end{array}$ \\
\hline \multicolumn{7}{|l|}{ Panel B. Hospitalizations by age in days } \\
\hline $\begin{array}{l}\text { Birth hospitalizations } \\
\quad(0 \text { days at admission })\end{array}$ & 238,790 & 0.0967 & $\begin{array}{c}0.0036 \\
(0.003)\end{array}$ & $\begin{array}{c}0.0016 \\
(0.002)\end{array}$ & $\begin{array}{c}0.0003 \\
(0.002)\end{array}$ & $\begin{array}{c}-0.0014 \\
(0.001)\end{array}$ \\
\hline First week hospitalizations (1-7 days) & 124,977 & 0.0517 & $\begin{array}{c}-0.0005 \\
(0.002)\end{array}$ & $\begin{array}{c}0.0014 \\
(0.001)\end{array}$ & $\begin{array}{c}0.0016 \\
(0.001)\end{array}$ & $\begin{array}{r}0.0007 \\
(0.001)\end{array}$ \\
\hline $\begin{array}{l}\text { Second and third week } \\
\text { hospitalizations ( } 8-21 \text { days })\end{array}$ & 46,112 & 0.0189 & $\begin{array}{l}0.0030 * * \\
(0.001)\end{array}$ & $\begin{array}{l}=0.0033^{* * * *} \\
(0.001)\end{array}$ & $\begin{array}{l}0.0037 * * * \\
(0.001)\end{array}$ & $\begin{array}{l}0.0030 * * * \\
(0.001)\end{array}$ \\
\hline
\end{tabular}

Notes: Each coefficient comes from a different regression. An observation is a newborn baby. The sample includes all babies born in the last one to four weeks of October, December, and February or the first one to four weeks of November, January, and March (depending on the column), for October-March sets from 2000-2001 to 2011-2012. The coefficients correspond to a binary explanatory variable indicating December 2010-January 2011 births (the weeks right around benefit cancellation). Control variables include month and year fixed effects. Robust standard errors are shown in parentheses. The number of observations in the four-week window is $2,553,272$.

*** Significant at the 1 percent level.

** Significant at the 5 percent level.

* Significant at the 10 percent level.

a result of the benefit cancellation. Given that there are about 1.9 hospitalizations per 100 children in this age range, this result implies a staggering 17 percent increase in the hospitalization rate in this age range.

\section{Conclusion}

Infant health has been shown to have important long-term consequences on human capital formation as well as adult health. Many births are scheduled early for nonmedical reasons. Our results suggest that scheduling birth early for nonmedical reasons may have negative health effects for the newborn, and these effects could be persistent. Our data will allow us to learn more about these effects by analyzing the specific diagnoses driving the estimated increases in hospitalization rates, as well as the extent to which the negative health consequences of early delivery persist over time.

Our findings imply that reducing the increasingly common practice of scheduling birth early for nonmedical reasons, even for term pregnancies, could prove an effective way of improving infant health, with potentially far-reaching effects.

\section{REFERENCES}

Borra, Cristina, Libertad González, and Almudena Sevilla. 2015. "The Impact of Scheduling Birth Early on Infant Health." Barcelona GSE Working Paper 707.

- Boyle, Elaine M., Gry Poulsen, David J. Field, Jennifer J. Kurinczuc, Dieter Wolke, Zarco Alfirevic, and Maria A. Quigley. 2012. "Effects of gestational age at birth on health outcomes at 3 and 5 years of age: population based cohort study." British Medical Journal 344: e896.

Byanova, Desislava. 2015. "Is It Worth the Wait? Early Elective Deliveries, Procedure Use, and Neonatal Health.” Unpublished.

-Fletcher, Jason M., Jeremy C. Green, and Matthew J. Neidell. 2010. "Long Term Effects of Childhood Asthma on Adult Health." Journal of Health Economics 29 (3): 377-87.

-Schulkind, Lisa, and Teny Maghakian Shapiro. 2014. "What a Difference a Day Makes: Quantifying the Effects of Birth Timing Manipulation on Infant Health." Journal of Health Economics 33: 139-58.

-Smith, James P. 2009. "The Impact of Childhood Health on Adult Labor Market Outcomes." Review of Economics and Statistics 91 (3): 478-89. 
This article has been cited by:

1. Kasey Buckles, Melanie Guldi. 2017. Worth the Wait? The Effect of Early Term Birth on Maternal and Infant Health. Journal of Policy Analysis and Management 36:4, 748-772. [Crossref] 